$\xi \mathrm{p}=$

\title{
Perceived stress among nurses of psychiatric ward patients with and without suicidal behavior
}

\author{
Ali H. Haider *, Uzma Masroor \\ Department of Clinical and Professional Psychology, Bahria University \\ *Corresponding author E-mail: psychobreath@gmail.com
}

\begin{abstract}
Background and Objectives: The present research was conducted to obtain a perceived stress pattern among nurses attending the psychiatric patients with suicidal and non-suicidal behavior in the different hospitals and clinics of the twin- cities (Islamabad and Rawalpindi) of Pakistan. The main objective of the study was to have an idea about the environment of nursing in psychiatric wards in Pakistani culture.

Participants and Method: The sample $(\mathrm{N}=36)$ comprised of the nurses of psychiatric ward attending the one patient for 12 hours $(n=16)$ with and $(n=20)$ without suicidal behavior working into night and day shifts respectively. All the participants gave responses on the perceived stress scale. Participants were approached during their duty hours and were given perceived stress scale 10- items along with demographic information sheet and the consent form. Between group-design was used in the present study.

Results and Conclusion: The results indicated that verification of both hypotheses, i.e. the nurses of patients with suicidal behavior had more perceived stress $(M=42 \pm 3.5)$ than the nurses of patients without suicidal behavior $(M=19.11 \pm 5.7)$ and also the old nurses had a greater level of perceived stress than the younger nurses according to the same statistics. The $t-$ test, $t(28.5)=14.9 \mathrm{p}<0.05$ was significant on $95 \%$ confidence interval with variance not equal. Limitations and suggestions for future were also discussed. Both the hypotheses were supported by the analysis- results and by the previous literature.
\end{abstract}

Keywords: Nurses; Perceived Stress; Psychiatric Patients; Suicidal \& Non Suicidal Behavior.

\section{Introduction}

The perceived stress is more intense than the actual stress. The most vulnerable population usually is of the professionals dealing with emergency health and psychological issues in hospitals. Moreover, the nurses appear more indulged in terms of their direct working contact hours with patients. The Perceived stress in nurses usually comes from the patients they attend in the hospitals and clinical settings, and these patients may have a mild to severe level of disorders with or without suicidal behavior. The perceived stress manifests itself in the form of burnout, health issues, and psychological reactions and specially emerges in nurses attending the patients with and without suicidal ideation and attempt. Pakistan is located in South Asia as Islamic country having a gigantic population of 196 million. Being an Islamic country, there is much influence of religion on beliefs of native human beings that attempted suicide or had an idea of suicide; both these beliefs are forbidden by their religion. On the other hand, there are a lot of suicide attempts despite all the religious or judicial barriers which are never reported in any Pakistani community (Ali \& Prince 2003). The possible rationale for the present study was that if a Pakistani research done on the nurses revealed the high occupational stress among nurses of psychiatric ward patients (Khalid \& Faisal 2010), then, the perceived stress also might have been present among nurses of psychiatric ward patients in Pakistan.

Hans Selye (1974), a Canadian renowned stress theorist chronicled stress as nonspecific responses throughout the body to any demand made upon it in three phases of "alarm" when body gets a sensation of something, the resistance phase where body's im- mune system uses adaptation energy to cope with the sensation. If this energy is not sufficient, the body goes in the third phase known as exhaustion and this is a stage of perceived stress, and the theory is known as General Adaptation Syndrome (p. 27). When the environment turns into a place where the psychiatric ward patients are provided with care by the Nurses, that environment is termed as "The Ward Atmosphere" (Beazley \& Gudjonsson 2011, Brunt 2008, Johansson \& Eklund 2004). Some other terms include treatment environment (Burti \& Mazzi 2004, Hawthorne et al. 2009, Røssberg et al. 2008), treatment climate (Timko \& Moos 1998), treatment milieu (Pedersen \& Karterud 2007), psychosocial environment (Brunt \& Hansson 2002), and healthcare environment (Johansson et al. 2006). Many of the researches support only for the importance of Ward atmosphere but the mental states of nurses is not emphasized in terms of understanding and management. The nurses are prone to perceived stress from the intense psychiatric environment.

The previous literature was based on a number of researches on nursing students, nurses and stress among nurses. Few researchers were based on the perceived stress among nurses of emergency wards, intensive care units and psychiatric wards (V. J. C. McCarthy \& B. A. Greiner 2010). The diploma holder nurses in Ireland under a research program revealed that "to crammer the theory material to get it stuck in mind really makes that stuff to get entangled and amalgamated in the mind waves which led them to a mal functioning clinical practice during their later practical profession and that became a leading source of perceived stress among nurses (Evans \& Kelly 2004). Ipso facto, the immaturity of the nursing skills and the practice blunders in the clinical set up are the major perceived stress producers in the psychiatric wards. 
Nurses may undergo a pressure of non-assertive threatening type of a relationship in day to day communication with their teaching staff that is a major source of perceived stress among them (Kleehammer et al. 1990). Nurses reported some negative views about their teaching staff by putting the allegations of being inquisitive all the time, requiring extra hard work, being the blackmailers and being harder over their internee nurses (Mahat 1996). A year later, the Irish nurses doing diploma in nursing profession, reported the same feelings of stress in another research (Evans \& Kelly 2004). Moreover, a sense of being ignored for not being observed by their seniors, the overloaded working shifts with fix time limitations, sense of being a minute part of a whole big clinical environment with certain kind of jobs to do and having a feeling of being insecure about their nursing tenure in the same clinical setup were the havocs of stress among the psychiatric nurses in Hong Kong (Keltner \& Leung 1995). However, little is known about the perceived stress trends in Pakistani Nurses. Nurses who are senior and have more expertise than the junior ones having less expertise show a livelier and a sympathetic attitude while talking with suicide patients (McLaughlin 1994, Samuelsson 1997). The caregivers for the psychiatric patients in Pakistani hospitals are mostly nurses, and their large population goes through depression \& anxiety. If the nurses provide a better care for the patients, they attend, the well-being of their patients is likely to increase and if the quality of the care is not up to the significant satisfactory level, then the patients may cause a dangerous level of stress for their caregivers, and this stress may affect the working capabilities of the caregivers (Tabassum. An et al. 2014). There is always a high expectancy from the nurses of mental health departments in almost all the hospital/ clinical wards to protect their patients from being dead and to keep every danger away from them in order to keep them safe and alive as it is included in the professional responsibilities of the nurses and when they perform their duties in the ward of suicidal patients, they mostly go through a stress while thinking upon all the possible ways to keep a patient alive with already having a tendency to suicide or with a desire not to be alive. This thinking leads them to a higher clash of behavior with their patients who ultimately affects the care providing quality and leads the nurses to a higher level of perceived stress (Whitworth 1984). The rude behavior of nurses to their patients makes their patients think that they are being humiliated by the nurse, and they perceive it as their nurses also want to get rid of them (Lewis et al. 1986). Nurses have a senseless attitude towards the patients of suicidal behavior when they meet these patients for the very first time besides the fact that these patients are at the urge to commit a suicide (Goldney \& Bottrill 1980).

\subsection{Purpose of the study}

This research, for the very first-time aims at determining the perceived stress level not in adolescents but in the young and old adult nurses in the age range of 18- 65, to get the nurses of psychiatrical wards on board and to get a better clinical view of the stress levels created by the patients of psychiatrical wards in their Nurses, to find out the severity of stress due to suicidal behavior and to comparison the stress levels with and without suicidal behavior in psychiatrical wards, to have an idea about the environment of nursing in psychiatrical wards in Pakistani culture and to have a reliability estimate of the used instrument of Perceived Stress for the current study (the psychometric properties for the Pakistani culture were already defined by the authors of Urdu translated version of the scale).

\subsection{Hypotheses}

The hypotheses for the study were proposed by me.

1) "I proposed that nurses attending the patients with suicidal behavior have been higher level of perceived stress than the nurses attending the patients without suicidal behavior."

2) "I proposed that nurses of older age have high perceived stress level than the younger nurses".

\section{Participants and method}

\subsection{Sample}

The nurses attending the psychiatrical ward patients with or without suicidal behavior from the different hospitals and from some clinics in twin cities of the Pakistan were taken as a sample for the current study $(\mathrm{N}=36)$. All the nurses were of the psychiatrical wards with age range of 25- 65 with regard of inclusion and exclusion criteria. Because it was a convenient sampling, so G- Power analysis for sample size was not done as it would have of no particular worth for the present sample.

\subsection{Design}

The research design for the present study was descriptive comparative as the perceived stress scale (10- items) was used to measure the stress levels in categories comprising old, young, and nurses of psychiatric wards with and without special diploma for suicidal care, and a comparison was made between all these stratified categories.

\subsection{Measure}

The Perceive stresses Scale (Cohen Sheldon 1943) Urdu translated Version (Amir K et al. 2011). This scale contained 10 items in total. The four items $(4,5,7$ and 8$)$ were positively stated about stress and the rest of the 6 items $(1,2,3,6,9$ and 10) with reverse scoring were negatively stated on stress. The responses were on 5 itemed likert-type scale, including $(1=$ never, $2=$ almost never, $3=$ sometimes, $4=$ fairly often and $5=$ very often). The same scale was used throughout the study in Urdu translated scale by ms. Aniqa Mariyam (the Urdu- translator of the scale from International Islamic University Islamabad). The PSS score range was from 0 56; scores might be computed after reversing the scoring (items 4 , 5, 7 and 8), higher scores indicated the greater perceived stress (Cohen et al., 1943). The PSS has exhibited satisfactory internal consistency (Cronbach's alpha 0.85) and good test- retest reliability (0.85) over two days (Kalaldeh \& Shosha 2012) in college student samples (Chao 2012, Cohen et al. 1983, Mitchel et al. 2008).

\subsection{Procedure}

In the informed consent, there was a brief mention about the confidentiality of the data and about the consent of the nurses to participate in data. Below was the demographic sheet with variables of name, age, occupation, qualification, condition of the patient they took care of (with suicidal behavior or without suicidal behavior) and the duty shifts (day or night shift). The informed consent contained the agreement from the Nurses whom they are participating in the research with their own will. The permission request letters signed by the HOD of the clinical psychology department of the Bahria University Islamabad were obtained with the help of respective student coordinator of the department. Those permission request letters were submitted in the offices of the HOD Doctors of the respective hospitals in the twin cities (Islamabad \& Rawalpindi).

\section{Results}

Table 1 revealed about the demographics that 36 nurses completed the given questionnaire. The mean age of the nurses was $35.92 \pm$ 16.92 with the age range of $18-64$. All the participants were female (nurses). The $56 \%$ nurses were of patients without suicidal behavior and they had a qualification level of General Nursing Diploma. On the same note, $44 \%$ nurses were attending the patients with suicidal behavior and they had a qualification level of Special Nursing Diploma. The nurses who participated in the research had an age range of 18-64. 
Table 1: Demographic Sheet Results

\begin{tabular}{|c|c|c|c|c|c|c|}
\hline S.n & Characteristics & $\mathrm{N}$ & $\%$ & Mean & SD & Range \\
\hline 1 & Occupation (Nursing) & 36 & 100 & & & \\
\hline \multirow[t]{2}{*}{2} & Qualification & & & & & \\
\hline & Nurses with General Nursing Diploma & 20 & 56 & & & \\
\hline \multirow[t]{3}{*}{3} & Nurses Caring for & & & & & \\
\hline & Patients(with suicidal behavior) & 16 & 44 & & & \\
\hline & Patients (without suicidal behavior) & 20 & 56 & & & \\
\hline 4 & Age & & & 35.64 & 16.92 & $18-64$ \\
\hline
\end{tabular}

There were no missing values detected in the demographics.

Table 2: Descriptive Data

\begin{tabular}{|c|c|c|c|c|c|c|c|c|}
\hline \multirow[b]{2}{*}{ Variables } & \multirow[b]{2}{*}{ Mean } & \multirow[b]{2}{*}{$\alpha$} & \multirow[b]{2}{*}{ SD } & \multicolumn{2}{|c|}{ Skewness } & \multicolumn{2}{|c|}{ Kurtosis } & \multirow{2}{*}{$\begin{array}{l}95 \%(\mathrm{CI}) \\
\text { Range } \\
\text { LL- UL }\end{array}$} \\
\hline & & & & Value & Std. Error & Value & Std. Error & \\
\hline Perceived Stress & 30.6 & .97 & 12.5 & -0.18 & 0.39 & -1.5 & .77 & $10-50$ \\
\hline Age & 35.6 & & 16.9 & .467 & .393 & -1.48 & .79 & $18-64$ \\
\hline
\end{tabular}

The significance of the Perceive Stress i.e. $\mathrm{p}<.05$, so the data according to \pm 2 (in terms of kurtosis for univariate) was normally distributed along the Mean (Perceived Stress $M=30.56$ ) and there was no need of any \pm 3 formula or $+10 \log$ formula in $\mathrm{z}$ - scores. In Table 3 the comparison of the extreme values showed that the old nurses attending the patients of suicidal behavior had a range of 14 i.e. $(50-36=14)$ and the young nurses attending the patients without suicidal behavior had a range of 20 i.e. $(30-10=20)$ for perceived stress scores. The less range of perceived stress for the nurses dealing with patients of suicide denoted that these nurses had a high level of stress, and the age distribution confirmed that these nurses were older than the nurses of the second category. The range difference $(20-14=6)$ indicated that the perceived stress level in the older nurses was starting from a higher level of 6 degrees than those of the young nurses attending patients without suicidal behavior.

Table 3: Extreme Values of Perceived Stress

\begin{tabular}{|c|c|c|c|c|c|c|c|}
\hline \multicolumn{4}{|c|}{$\begin{array}{l}\text { Perceived Stress Extreme Values } \\
\text { Suicide }\end{array}$} & \multicolumn{4}{|c|}{ Without Suicide } \\
\hline $\begin{array}{l}\text { High- } \\
\text { Case\#'s }\end{array}$ & $\begin{array}{l}\text { Val } \\
\text { ue }\end{array}$ & $\begin{array}{l}\text { Low- } \\
\text { Case } \\
\text { \#'s }\end{array}$ & $\begin{array}{l}\text { Val } \\
\text { ue }\end{array}$ & $\begin{array}{l}\text { High - } \\
\text { Case \#'s }\end{array}$ & $\begin{array}{l}\text { Val } \\
\text { ue }\end{array}$ & $\begin{array}{l}\text { Low - } \\
\text { Case \#'s }\end{array}$ & $\begin{array}{l}\text { Val } \\
\text { ue }\end{array}$ \\
\hline 4 & 50 & 1 & 36 & 35 & 30 & 20 & 10 \\
\hline 8 & 46 & 5 & 38 & 27 & 26 & 24 & 10 \\
\hline 10 & 46 & 14 & 39 & 21 & 25 & 26 & 12 \\
\hline 13 & 46 & 12 & 39 & 19 & 24 & 22 & 13 \\
\hline 11 & 45 & 3 & 39 & 28 & 23 & 32 & 14 \\
\hline
\end{tabular}

These case numbers are responsible for the lowest and highest values of perceived stress in the referring categories of comparison for perceived stress scores.

Statistics for t- Test Groups: Table 3.P. contained the Suicidewithout Suicide variable statistics for perceived stress scores and this analysis showed equal number of participants $(\mathrm{N}=18)$ for both the groups.

Table 4: Group Statistics T- Test

\begin{tabular}{lllll}
\multicolumn{5}{c}{ Table 4: Group Statistics T- Test } \\
\hline \multicolumn{5}{c}{ Perceived Stress in } \\
\hline Nurses \\
Perceived Stress in Nurses & $\mathrm{N}$ & $\mathrm{M}$ & $\mathrm{SD}$ & $\begin{array}{l}\text { Std. Error } \\
\text { Mean }\end{array}$ \\
$\begin{array}{l}\text { Nurses of suicide patients } \\
\begin{array}{l}\text { Nurses f non- suicide pa- } \\
\text { tients }\end{array}\end{array}$ & 14 & 42 & 3.51 & 0.83 \\
\hline
\end{tabular}

The group containing nurses of nurses attending patients with suicidal behavior had a mean perceived stress score $40(M=40 \pm$ 3.51 ), scored high on the perceived stress scale and the group containing nurses attending the patients without suicidal behavior having a mean perceived stress score $19(\mathrm{M}=19 \pm 5.7)$, scored low on the perceived stress scale.

Independent Sample t- test

\begin{tabular}{llllllll}
\multicolumn{10}{c}{ Table 5: Independent Sample T- Test Analysis } \\
\hline & \multicolumn{1}{c}{$\begin{array}{l}\text { 95 \% CI of the } \\
\text { Difference }\end{array}$} \\
\hline & t & Df & $\begin{array}{l}\mathrm{p}(2- \\
\text { tailed })\end{array}$ & $\begin{array}{l}\text { M } \\
\text { D }\end{array}$ & $\begin{array}{l}\text { Std. Error } \\
\text { Difference }\end{array}$ & LL & UL \\
Perceived & 14 & 28 & 0.000 & 22 & 1.6 & 19.67 & 26.08 \\
Stress & .5 & .3 & & .9 & & & \\
\hline
\end{tabular}

The nurses of suicide patients experienced high perceived stress $(\mathrm{M}=42, \mathrm{SE}=.83)$ than to the nurses of patients without suicide $(\mathrm{M}=19.11, \mathrm{SE}=1.35)$. The significant $\mathrm{t}=14.5$ showed $\mathrm{t}(28.3)=$ 14.5, p < .05; sig. $0.03<.05$ at 2 tailed sig and the effect size $(14.5) 2 /(14.5) 2+28.3=.88$ showed a high value i.e. the effect size was high.

\section{Discussion and conclusion}

The first hypothesis was proved by the statistics of this study as 18- 30 was a low-range score for nurses attending suicidal patients as compared with 35- 50 high range score of nurses attending patients without suicidal behavior. The previous literature in support of the current hypothesis spoke as the nurses attending the patients with suicidal behavior do not try to make a verbal connection with them (Joyce 2003) as many patients of suicidal behavior raise a complaint that when nurses attend them, they have not been able to make a vocal connection to them as nurses dislike doing so (Fletcher 1999, Jones et al. 2001). The nurses reported a high level of wrath, dismay, some phobic behavior (because of attending patients with suicidal behavior) and some stress of being upset emotionally and some aggressive impulsivity. The arrival of a patient on the level where they attempt to commit suicide during the treatment process leads the nurses to a high level of depression and stressful state of mind (Joyce, 2003). A document of public domain and permitted to be reported freely) published in July 2008, by National institute of Occupational Safety and health (Department of Health and Human Services) stated the two basic reasons of stress among nurses, i.e. number one was the role conflict in decision-making power of nurses about their patients illness and the second was "attending the psychiatric patients with more serious illnesses (clinical depression, severe anxiety and patients of suicidal behavior)". All these statements from the different researches are enough to support the proven $1^{\text {st }}$ hypothesis of the current study.

The second hypothesis was also proved by the current study research analysis which showed the age range of young nurses 1824 and for the older, nurse was 29- 64. For the range of the young nurses, the range of perceived stress score remained in the range 10- 30 and for the older nurses, the perceived stress range went to 36- 50. By this range, it was obvious that the older nurses had more perceived stress than the younger nurses.

The previous literature supported the proven hypothesis as the older nurses might have seen more psychiatric suicidal behaviors 
in the patients for several times on a really serious level (Bultema $1994 \&$ Cooper 1995). The stress of workplace also dominates in the older nurses (Andrews et al. 2005).The The work performance goes down with the ageing process and the ability to handle the situation also decreases with it in older nurses than in younger nurses (Alpass \& Mortimer 2007, Hatcher et al. 2006, Letvak 2002). The correlation of ageing and stress among nurses came out to be significant $(p<.05)$, and the nurses of age range 51-60 retained a highest stress level than the nurses of young age range from19-30 which retained the lowest level of stress $(55.6 \%$ and25.9\% respectively) was a statistical analysis in a research "Work Related Stress Among Nurses: A Challenge for Health Care Institution (Bettina Piko et al., 1999) and these are the enough proofs to support the second proven hypothesis of the current research.

Both the hypotheses were supported by the analysis- results and by the previous literatures. A high perceived stress was reported among the nurses of psychiatric wards dealing patients with suicidal behavior. In the present thesis, the aim was to have a perceived stress level among the nurses of psychiatrical wards attending the patients of suicidal behavior and the patients without suicidal behavior in a night and day shifts respectively, and that was attained successfully.

\subsection{Limitations}

There are a few limitations as well regarding the scale, the data collection process, the target population and about some timemanagement issues for the thesis given here. The Perceived Stress Scale was not a diagnostic instrument; there were no score cutoffs. There were only comparisons within the sample. The normative form contains only a narrow range of normative samples. The confirmatory analysis was not performed as it wasn't a social study, and also the regression analysis could not have been done on the data variables. This scale only measured the perceived stress only for previous one month. Only the perceived stress was being measured in the nurses and the study remained limited about having a quick measure of coping strategies nurses used to cope stress because of the limited space for adding more variables as the approval for the chosen population was already really difficult and if the questionnaire items would have increased by adding one more scale, the participants might have easily refused to participate and research might not have been possible.

\section{References}

[1] Asghari, F., Sadeghi, A., Aslani, K., Saadat, S., \& Khodayari, H (2013). The Survey of Relationship between Perceived Stress, Coping Strategies and Suicide Ideation among Students at University of Guilan, Iran. International Journal of Education and Research, 1 (11), 111-118.

[2] Armitage, G. C. (2004). Periodontal diagnoses and classification of periodontal diseases. Periodontology 2000, 34(1), 9-21. https://doi.org/10.1046/j.0906-6713.2002.003421.x.

[3] Brandtstädter, J., \& Renner, G. (1990). Tenacious goal pursuit and flexible goal adjustment: explication and age-related analysis of assimilative and accommodative strategies of coping. Psychology and aging, 5 (1), 58. https://doi.org/10.1037/0882-7974.5.1.58.

[4] Bartrop, R. W., Lazarus, L., Luckhurst, E., Kiloh, L. G., \& Penny, R. (1977). Depressed lymphocyte function after bereavement. The Lancet, $\quad 309(8016), \quad 834-836 . \quad$ https://doi.org/10.1016/S01406736(77)92780-5.

[5] Cohen, S. (1988). Perceived stress in a probability sample of the United States.

[6] Dubow, E. F., Kausch, D. F., Blum, M. C., Reed, J., \& Bush, E. (1989). Correlates of suicidal ideation and attempts in a community sample of junior high and high school students. Journal of Clinical Child Psychology, 18(2), 158-166. https://doi.org/10.1207/s15374424jccp1802_7.

[7] Hawthorne, W. B., Green, E. E., Folsom, D., \& Lohr, J. B. (2009) A randomized study comparing the treatment environment in alternative and hospital-based acute psychiatric care. Psychiatric Ser vices. https://doi.org/10.1176/ps.2009.60.9.1239.
[8] Irfan, u., sheikh, s., \& faisal, m. (2011). Frequency of depression due to stress in female nurses.Khyber medical university journal, 2(1), 10-14.

[9] Jyotsna, V. P., Ambekar, S., Singla, R., Joshi, A., Dhawan, A., Kumar, N., \& Sreenivas, V. (2013). Cardiac autonomic function in patients with diabetes improves with practice of comprehensive yogic breathing program. Indian journal of endocrinology and metabolism, 17(3), 480. https://doi.org/10.4103/2230-8210.111645.

[10] Khalid, S., Irfan, U., Sheikh, S., \& Faisal, M. (2010). Frequency of stress and depression in female nurses working in a teaching hospital. Kust Med J, 2, 10-14.

[11] Khan, m. M., \& ali hyder, a. (2006). Suicides in the developing world: case study from pakistan.

[12] Suicide and life-threatening behavior, 36(1), 76-81. https://doi.org/10.1521/suli.2006.36.1.76.

[13] Kendler, K. S., Kessler, R. C., Walters, E. E., MacLean, C., Neale, M. C., Heath, A. C., \& Eaves, L. J. (2010). Stressful life events, genetic liability, and onset of an episode of major depression in women. Focus, 8(3), 459-470. https://doi.org/10.1176/foc.8.3.foc459.

[14] Nock, M. K., Hwang, I., Sampson, N. A., \& Kessler, R. C. (2010). Mental disorders, comorbidity and suicidal behavior: results from the National Comorbidity Survey Replication. Molecular psychiatry, 15(8), 868-876. https://doi.org/10.1038/mp.2009.29.

[15] Nuovo, S. (2006). Psychological stress measure in type 2 diabetes. European review for medicaland pharmacological sciences, 10(2), 69.

[16] Ratnakant, S., Ochs, M. E., \& Solomon, S. S. (2003). Sounding board: diabetes mellitus in the elderly: a truly heterogeneous entity? Diabetes, Obesity and Metabolism, 5 (2), 81-92. https://doi.org/10.1046/j.1463-1326.2003.00242.x.

[17] Scales. (n.d.). $\quad$ Retrieved from http://www.psy.cmu.edu/ scohen/scales.html

[18] Selye, H. (1950). Stress and the general adaptation syndrome. British medical journal, 1 (4667), 1383. https://doi.org/10.1136/bmj.1.4667.1383.

[19] Sidhartha, T., \& Jena, S. (2006). Suicidal behaviors in adolescents The Indian Journal of Pediatrics, 73 (9), 783-788. https://doi.org/10.1007/BF02790385.

[20] Selye, H. (1974). Stress sans détresse. Lippincott. Selye, H. (1956). The stress of life.

[21] Tanuj, S., \& Shivananda, J. Suicidal behaviors in adolescents.

[22] Tanaka, E., Sakamoto, S., Kijima, N., \& Kitamura, T. (1998). Different personalities between depression and anxiety. Journal of clinical psychology, $54 \quad$ (8), 1043-1051. https://doi.org/10.1002/(SICI)1097-4679(199812)54:8<1043::AIDJCLP4>3.0.CO;2-A.

[23] Tellegen, A. (1985). Structures of mood and personality and their relevance to assessing anxiety, with an emphasis on self-report.

[24] Trovato, g. M., catalano, d., martines, g. F., spadaro, d., di corrado, d., crispi, v., \& di nuovo, s. (2006). Psychological stress measure in type 2 diabetes. European review for medical and pharmacological sciences, 10(2), 69.

[25] Wilcox, H. C., Arria, A. M., Caldeira, K. M., Vincent, K. B., Pinchevsky, G. M., \& O'Grady, K. E. (2010). Prevalence and predictors of persistent suicide ideation, plans, and attempts during college. Journal of affective disorders, 127 (1), 287-294. https://doi.org/10.1016/j.jad.2010.04.017.

[26] Wichstrøm, L. (2000). Predictors of adolescent suicide attempts: a nationally representative longitudinal study of Norwegian adolescents. Journal of the American Academy of Child \& Adolescent Psychiatry, 39 (5), 603-610. https://doi.org/10.1097/00004583200005000-00014. 